\title{
Segmentation techniques used image reconigation and SAR Image Processing
}

\author{
Joyjit Patra ${ }^{1}$,Arun Kanti Manna ${ }^{2}$,Himadri Nath Moulick ${ }^{3}$ and Ashish kumar ${ }^{4}$ \\ ${ }^{I}$ (Asst.Prof.In CSE.Dept Of Aryabhatta Institute Of Engineering And Management,Durgapur,W.B.India). \\ ${ }_{2}^{2}$ (Persuing Ph.D.From Techno India University,W.B.India.) \\ ${ }^{3}$ (Asst.Prof.In CSE.Dept Of Aryabhatta Institute Of Engineering And Management,Durgapur,W.B.India.) \\ ${ }^{4}\left(4^{\text {th }}\right.$ year Student,IT. Dept Of Aryabhatta Institute Of Engineering And Management,Durgapur, W.B.India.)
}

\begin{abstract}
Image segmentation is the process of partitioning an image intomultiple segments, so as to change the representation of an image into something that is more meaningful and easier to analyze.Several generalpurpose algorithms and techniques have beendeveloped for image segmentation.This paper describes the different segmentation techniques used in the field of ultrasound and SAR Image Processing. Firstly this paper investigates and compiles some of the technologies used for image segmentation.Then a bibliographical survey of current segmentation techniques is given in this paper and finally general tendencies in image segmentation are presented.
\end{abstract}

Keywords: Gray Histogram Technique, Pareto Approach (PTA),Segmentation,Clustering.

\section{INTRODUCTION}

Images are considered as one of the most important medium of conveying information,in the field of computer vision,by understanding images the information extracted from them can be used for other tasks for example: navigation of robots, extracting malign tissues from body scans, detection of cancerous cells,identification of an airport from remote sensing data.Now there is a need of a method, with the help of which,we can understand images and extract information or objects,image segmentation fulfill above requirements.Thus,image segmentation is the first step in image analysis.[27] ome time image denoising is done before the segmentation to avoid from the false contour selection for segmentation to segment the image without loss of information for medical diagnosing purpose is a challenging job.[16]The purpose of writing this paper is to provide a literature review in this arena.[19]The remainder of this paper is organized as below; section II introduces the term image segmentation. SectionIII describes the current image segmentation techniques and sectionIV Concludes the overall study.[10]

\section{IMAGE SEGMENTATION}

Image segmentation refers to the process of partitioning a digital image into multiple segments i.e. set of pixels,pixels in a region are similar according to some homogeneity criteria such as colour,intensity or texture, so as to locate and identify objects and boundaries in an image . Practical application of image segmentation range from filtering of noisy images,medical applications (Locate tumors and other pathologies,Measure tissue volumes,Computer guided surgery, Diagnosis,Treatment planning,study of anatomical structure),Locate objects in satellite images (roads, forests, etc.),FaceRecognition,Finger print Recognition,etc.Many segmentation methods have been proposed in the literature.[21]The choice of a segmentation technique over another and the level of segmentation are decided by the particular type of image and characteristics of the problem beingCo nsidered.

\section{CURRENT SEGMENTATION TECHNIQUES}

The Research on Image segmentation for many years has been a high degree of attention. Thousands of different segmentation techniques are present in the literature, but there is not a single method which can be considered good for different images,all methods are not equally good for a particular type of image.Thus, algorithm development for one class of image may not always be applied to other class of images.Hence,there are many challenging issues like development of a unified approach to image segmentation which can be applied to all type of images,even the selection of an appropriate technique for a specific type of image is a difficult problem.[8]Thus,in spite of several decades of research,there is no universally accepted method for image segmentation and therefore it remains a challenging problem in image processing and computer vision. Based on different technologies,image segmentation approaches are currently divided into following categories, based on two properties of image. 


\section{DetectingDiscontinuities}

It means to partition an image based on abrupt changes in intensity, this in cludes image segmentation algorithms like edge detection.[23]

\section{$>$ Detecting Similarities}

It means to partition an image into regions that are similar according to a set of predefined criterion;this includes image segmentation algorithms like thresholding, region growing,region splitting and merging.[1]

\section{SEGMENTATION BASED ON EDGE DETECTION}

This method attempts to resolve image segmentation by detectingthe edges or pixels between different regions that have rapid transition in intensity are extracted and linked to form closed object boundaries.[7]The result is a binary image.Based on theory there are two main edge based segmentation methods- gray histogram and gradient based method.

\section{$>$ Gray Histogram Technique}

The result of edge detection technique depends mainly on selection of threshold $\mathrm{T}$, and it is really difficult to search for maximum and minimum gray level intensity because gray histogram is uneven for the impact of noise, thus we approximately substitute the curves of object and background with two conic Gaussian curves,

whose intersection is the valley of histogram. Threshold $\mathrm{T}$ is the gray value of intersection point of that valley.

\section{$>$ Gradient Based Method}

Gradient is the first derivative for image $\mathrm{f}(\mathrm{x}, \mathrm{y})$, when there is abrupt change in intensity near edge and there is little image noise,gradient based method works well. This method involves convolving gradient operators with the image. High value of the gradient magnitude is possible place of rapid transition between two different regions. These are edge pixels, they have to be linked to form closed boundaries of the regions.Common edge detection operators used in gradient based method are sobel operator,canny operator,Laplace operator, Laplacian of Gaussian(LOG)operator \& so on,canny is most promising one,but takes more time as compared to sobel operator.[11]Edge detection methods requires a balance between detecting accuracy and noise immunity in practice, if the level of detecting accuracy is too high, noise may bring in fake edges making the outline of images unreasonable and if the degree of noise immunity is too excessive,some parts of the image outline may get undetected and the position of objects may be mistaken.Thus,edge detection algorithms are suitable for images that are simple and noise-free as well often produce missing edges or extra edges on complex and noisy images.

\section{THRESHOLDING METHOD}

Image segmentation by thresholding is a simple but powerful approach for segmenting images having light objects on dark background.Thresholding technique is based on imagespace regions i.e. on characteristics of image.[4]Thresholding operation convert a multilevel image into a binary image i.e.,it choose a proper threshold $\mathrm{T}$,to divide image pixels into several regions and separate objects from background.Any pixel $(\mathrm{x}, \mathrm{y})$ is considered as a part of object if its intensity is greater than or equal to threshold value i.e., $f(x, y) \geq T$,else pixel belong to background.As per the selection of thresholding value,two types of thresholding methods are in existence,global and local thresholding.[6]When $\mathrm{T}$ is constant,the approach is called global thresholding otherwise it is called local thresholding.Global thresholding methods can fail when the background illumination is uneven.In local thresholding,multiple thresholds are used to compensate for uneven illumination.[17] Threshold selection is typically done interactively however,it is possible to derive automatic threshold selection algorithms.[9]Limitation of thresholding method is that,only two classes are generated and it cannot be applied to multichannel images.[5]In addition,thresholding does not take into account the spatial characteristics of an image due to this it is sensitive to noise,as both of these artifacts corrupt the histogram of the image,making separation more difficult.

\section{REGION BASED SEGMENTATION METHODS}

Compared to edge detection method, segmentation algorithms based on region are relatively simple and more immune to noise.[22]Edge based methods partition an image based on rapid changes in intensity near edges whereas region based methods,partition an image into regions that are similar according to a set of predefined criteria.[25]Segmentation algorithms based on region mainly include following methods: 


\section{$>$ Region Growing}

Region growing is a procedure that group's pixels in whole image into sub regions or larger regions based on predefined criterion.Region growing can be processed in four steps:-

(i)Select a group of seed pixels in original image.

(ii)Select a set of similarity criterion such as grey level intensity or color and set up a stopping rule.

(iii)Grow regions by appending to each seed those neighbouring pixels that have predefined properties similar to seed pixels.

(iv)Stop region growing when no more pixels met the criterion for inclusion in that region (i.e. Size, likeness between a candidate pixel \& pixel grown so far,shape of the region being grown)

\section{$>$ Region Splitting and Merging}

Rather than choosing seed points,user can divide an image into a set of arbitrary unconnected regions and then merge the regions in an attempt to satisfy the conditions of reasonable image segmentation.Region splitting and merging is usually implemented with theory based on quad tree data.[2]Let $\mathrm{R}$ represent the entire image region and select a predicate $\mathrm{Q}$

(i)We start with entire image if $\mathrm{Q}(\mathrm{R})=$ FALSE [1], we divide the image into quadrants, if $\mathrm{Q}$ is false for any quadrant that is, if $\mathrm{Q}(\mathrm{Ri})=\mathrm{FALSE}, \mathrm{We}$ subdivide the quadrants into sub quadrants and so on till no further splitting is possible.[16]

(ii)If only splitting is used,the final partition may contain adjacent regions with identical properties.This drawback can be remedied by allowing merging as well as splitting i.e.merge any adjacent regions $\mathrm{Rj} \& \mathrm{Rk}$ for which, $\mathrm{Q}(\mathrm{Rj} \mathrm{U} \mathrm{Rk})=\mathrm{TRUE}$

(iii)Stop when no further merging is possible.

\section{SEGMENTATION METHODS BASED ON PDE (PARTIAL DIFFERENTIAL EQUATION)}

Using a PDE based method \& solving the PDE equation by a numerical scheme one can segment the image.Image segmentation based on PDEs is mainly carried out by active contour model or snakes.This method was first introduced by Kassetal in 1987 Kass developed this method to find familiar objects in presence of noise and other ambiguities.[9]The central idea of snake is transforming a segmentation problem into a PDE framework. That is, the evolution of a given curve,surface or image is handled by PDEs and the solution of these PDEs is what we look forward to various methods for image segmentation are-snake, level set and Mumford-shah model.[6]

\section{$>$ Snakes}

Active contours or snakes are computer generated curves that move within the image to find object boundaries under the influence of internal and external forces.This procedure is as follows:-

(i)Snake is placed near the contour of Region Of Interest (ROI).

(ii)During an iterative process due to various internal and external forces within the image, the Snake is attracted towards the target.These forces control the shape and location of the snake within the image.

(iii)An energy function is constructed which consist of internal and external forces to measure the appropriateness of the Contour of ROI,Minimize the energy function (integral), which represents active contour's total energy.The internal forces are responsible for smoothness while the external forces guide the contours towards the contour of ROI.[3] Shortcoming of traditional snake is that,it require user interaction,which consists of determining the curve around the detected object,the energy function often converge to minimum local energy,so snake should be placed usually near the boundary of ROI,original snake algorithm is particularly sensitive to noise.More sensitive to the choice of its parameters and adaptively adjusts the parameters in an extremely complex process.The computational complexity of the algorithm is high.To solve these problems, number of researchers, have made various improvements to basic model,but the shortcoming of snake are still not overcome fundamentally.

\section{$>$ Level Set Model}

Many of the PDEs used in image processing are based on moving curves and surfaces with curvature based velocities.In this area,the level set method developed by Osher and Sethian was very influential and useful.The basic idea is to represent the curves or surfaces as the zero level set of a higher dimensional hyper surface. This technique not only provides more accurate numerical implementations but also handle topological change very easily.[6]It has several advantages;its stability and irrelevancy with topology,displays a great advantage to solve the problems of corner point producing,curve breaking and combining etc.Since the edgestopping function depends on the image gradient, only objects with edges defined by gradients can be segmented.Another disadvantage is that in practice,the edge-stopping function is never exactly zero at the edges, and so the curve may eventually pass through object boundaries. 


\section{Dumford Shah Model}

The Mumford-Shah model uses the global information of the image as the stopping criterion to segment the image.Mumford-shah takes advantage of the entire information of the image to result in the best image segmentation.

\section{C-V Model}

The basic idea is to look for a particular partition of a given image into two regions, one representing the objects to be detected \& other background.C-V model is not based on edge function,to stop the evolving curve on desired boundary.[9](There is no need to smooth initial image,even if it is noisy),the location of boundary are very well detected.It can detect objects whose boundary are not necessarily defined by gradient or very smooth boundaries.Starting with only one initial curve this model can automatically detect interior contours and it does not necessarily start around the objects to be detected.

\section{SEGMENTATION BASED ON ARTIFICIAL NEURAL NETWORK}

Neural Network based segmentation is totally different from conventional segmentation algorithms.In this,an image is firstly mapped into a Neural Network. Where every Neuron stands for a pixel,thus image segmentation problem is converted into energy minimization problem. The neural network was trained with training sample set in order to determine the connection and weights between nodes.[19]Then the new images were segmented with trained neural network, for example,we can extract image edges by using dynamic equations which direct the state of every neuron towards minimum energy defined by neural network.Neural network segmentation includes two important steps feature extraction and image segmentation based on neural network.Feature extraction is very crucial as it determines input data of neural network,firstly some features are extracted from the images,such that they become suitable for segmentation and then they were the input of the neural network.[3]All of the selected features compose of highly non-linear feature space of cluster boundary. Neural network based segmentation have three basic characteristics:-

(i)Highly parallel ability and fast computing capability,which makes it suitable for real time application.

(ii)Improve the segmentation results when the data deviates from the normal situation.
(iii)Robustness
making
it insensitive
to
noise.

(iv)Reduced requirement of expert intervention during the image segmentation process.However there are some drawbacks of neural networks based segmentation either,such as:-

(a)Some kind of segmentation information should be known beforehand.

(b)Initialization may influence the result of image

segmentation.

(c)Neural network should be trained using learning process beforehand,the period of training may be very long,and we should avoid overtraining at the same time.

\section{SEGMENTATION BASED ON CLUSTERING}

Clustering is an unsupervised learning task, where one needs to identify a finite set of categories known as clusters to classify pixels.Clustering use no training stages rather train themselves using available data. Clustering is mainly used when classes are known in advance.[8]A similarity criteria is defined between pixels, and then similar pixels are grouped together to form clusters. The grouping of pixels into clusters is based on the principle of maximizing the intra class similarity and maximizing the inter class similarity. [7]The quality of a clustering result depends on both the similarity measure used by the method and its implementation.Clustering algorithms are classified as hard clustering,k- means clustering, fuzzy clustering, etc.

\section{$>$ Hard Clustering}

Hard clustering assumes sharp boundaries between clusters;a pixel belongs to one and only one cluster.A popular and well known hard clustering algorithm is K-means clustering algorithm.K-means algorithm is a clustering technique to partition $\mathrm{n}$ pixels into $\mathrm{k}$ clusters, where $\mathrm{k}<\mathrm{n}$.K-means algorithm Developed by Mac Queen in 1965 and then refined by Hartigan and Wong in1979.K-means algorithm is a clustering technique, which classify pixels in an image into $\mathrm{K}$ number of clusters, where $\mathrm{K}$ is a positive integer,according to some similarity feature like grey level intensity of pixels and distance of pixel intensities,from centroid pixel intensity.The main advantages of this algorithm are its simplicity and low computational cost, which allow it to run efficiently on large data sets.[5]The main drawback is that: $\mathrm{K}$ the number of clusters must be determined,it does not yield the same result each time the algorithm is executed and the resulting clusters depend on the initial assignments of centroids. The process is as follow

(i)Randomly choose number of clusters K.

(ii)Compute the histogram of pixel intensities.

(iii)Randomly choose $\mathrm{K}$ pixels of different intensities as Centroids. 
(iv)Centroids are finding out by calculating mean of pixel values in a region and place Centroids as much far away from each other as possible.

(v)Now,compare a pixel to every Centroid and assign pixel to closest Centroid to form a cluster.

(vi)When all pixels have been assigned,initial clustering has been completed.

(vii)Recalculate position of Centroids in K clusters .

(viii)Repeat step $5 \& 6$, until Centroids no longer move.

(ix)Image separated into K clusters.

\section{$>$ Fuzzy Clustering}

In real time applications, one of the most difficult task in image analysis \& computer vision is to classify the pixel in an image correctly,when there is no crisp boundaries between objects in an image thus in order to address this difficulty,fuzzy clustering techniques are used.[11]Fuzzy clustering technique classify pixel values with great extent of accuracy \& it is basically suitable for decision oriented applications like tissue classification \& tumor detection etc. fuzzy clustering divides the input pixels into clusters or groups on the basis of some similarity criterion,such that similar pixels belong to same cluster.Similarity criterion used can be distance,connectivity,intensity. The resulting partition improves the understanding of human beings \& helps in a more informed decision making.The advantage of fuzzy system is that they are easy to understand,as the membership function partition the data-space properly.[12]Fuzzy clustering algorithms include FCM(fuzzy C means)algorithm,GK(Gustafson-Kessel),GMD(Gaussian mixture decomposition),FCV(Fuzzy C varieties),AFC,FCS, FCSS,FCQS, FCRS algorithm and etc,among all The FCM is the most accepted method since it can preserve much more information than other approaches.FCM assign pixels to each class by means of membership function.Let us supposeX $=(\mathrm{x} 1, \mathrm{x} 2, \mathrm{x} 3-------\mathrm{xn})$ Denotes an image with $\mathrm{N}$ pixels which is to be divided into $\mathrm{C}$ clusters, $\mathrm{FCM}$ follows an iterative process which minimize following objective function- $\mathrm{ijm} \| \mathrm{xj}$ - vi $\|$ Where,uijm $=$ membership function of pixel $\mathrm{xj}$ in ith cluster $\mathrm{Vi}$ is the centre pixel of ith cluster $\mathrm{m}$ is the fuzzifier that controls the fuzziness of resulting clusters \& lies between $1 \mathrm{~m} \leq \infty$ The membership function and cluster centers are updated,the cluster centers can either be initialized randomly or by an approximation method.[14] Disadvantage of FCM is that for noisy images it does not take into account spatial Information, which makes it sensitive to noise \& other image artifacts.As FCM cluster assignment is based on distribution of pixel intensity, it makes it sensitive to intensity variations in the illumination.[19]To overcome these drawbacks of FCM,several other algorithms are introduced as modified FCM,GSFCM(Generalized spatial FCM), mean shift based FCM,FLICM(fuzzy logic information C-means clustering algorithm),NFCM (novel FCM), ISFCM (improved spatial FCM).[20]

\section{MULTIOBJECTIVE IMAGE SEGMENTATION}

Earlier image segmentation problem has been treated as monoobjective.Mono-objective images considers only one objective,because a single segmentation image. Such type of segmented images are of good quality but may not allow a higher level process(as image segmentation considered as low level process \& pattern recognition, object tracking \& scene analysis as high level process) to extract all information included within the image.So different segmentation results are calculated.[15]Image segmentation is a multi objective optimization problem. The consideration of multiple criteria(objectives)starts from the understanding of image pattern to its selected segmentation process involved (feature selection/extraction,similarity/dissimilarity measure) and finally the assessment of its output(validity assessment). As there are possibilities of multiple sources of information for a segmentation problem,thus multiple representations have to be considered,for example,feature selection is the process of identifying similarity criteria used in segmentation process,now either only single criteria is used,that is intensity of pixels,or to make it a multiobjective problem consider several similarity criteria to segment same image, which can be intensity,colour,texture, shape,spatial information. For instance,in segmenting a medical image based on CT scan, multiple features like intensity,shape and spatial relationship could be considered.Similarly criteria for inter pattern similarity that is grouping can be multiple,spatial coherence vs. feature homogeneity,connectedness vs. compactness, diversity vs.accuracy.For image segmentation multiple methods can be used for getting appropriate output,and there may be a tendency for multiple optimizations and decision making processes where multiple validity assessment should be used.[24]There are two general approaches for Multiobjective optimization problem,the first approach is to combine multiple objective functions into a single composite function, and the second is to determine a set of solutions that are non-dominated with respect to each objective.

\section{The Conventional Weighted Formula Approach(WFA)}

In this approach a Multiobjective problem is Transformed into a problem with single objective,that is typically done by assigning a numerical weight to each objective and then combining the values of weighted 
criteria into a single value by either adding or multiplying weighted criteria.[26]The quality of a given candidate model is given by one of the two kinds of formula:

$\mathrm{Q}=\mathrm{w} 1 \mathrm{c} 1+\mathrm{w} 2 \mathrm{c} 2----------+\mathrm{wncn}$

$\mathrm{Q}=\mathrm{w} 1 \mathrm{c} 1 \times \mathrm{w} 2 \mathrm{c} 2----------\times$ wncn

$\mathrm{Wi}, \mathrm{i}=1,2,--------n$, denotes the weight assigned to criteria ci and $\mathrm{n}$ is the number of evaluation criteria.

\section{$>$ Pareto Approach (PTA)}

The basic idea is that, instead of transforming a Multiobjective problem into a single objective function, and then solving it by using a single objective search method,one uses a Multiobjective algorithm to solve the problem. The formulation starts with simultaneous optimization of several objectives, a reasonable solution is to investigate a set of solutions each of which satisfies the objectives at an acceptable level without being dominated by other solutions, these solutions are called non-dominated solutions and the region of those solutions is called Pareto front.[22]

\section{CONCLUSION}

In this paper we classify and discuss main image segmentation algorithms.Image segmentation has a promising future as the universal segmentation algorithm and has become the focus of contemporary research.[2]In spite of several decades of research up to now to the knowledge of authors, there is no universally accepted method for image segmentation, as the result of image segmentation is affected by lots of factors, such as: homogeneity of images,spatial characteristics of the image continuity,texture,image content.[4]Thus there is no single method which can be considered good for all type of images,nor all methods equally good for a particular type of image.Due to all above factors,image segmentation remains a challenging problem in image processing and computer vision and is still a pending problem in the world.

\section{Acknowledgements}

The authors are very thankful to Mr.Saikat Maity(Associate Professor Of Dr.B.C.Roy Engineering College) and Dr.chandan Koner(Associate Professor Of Dr.B.C.Roy Engineering College) for pepering this paper.

\section{REFERENCES}

[1] A. K. Jain, R. Duin, "Statistical pattern recognition: A review". IEEE Transactions on Pattern Analysis and Machine Intelligence, vol. 22, no.1, pp. 4-37, 2000.

[2] A. N. Otsu, "A threshold selection method from gray-level histogram". IEEE Transactions on Systems, Man. And Cybernetics, pp. 62-66, 1978

[3] T. A. Pun, "New method for gray-level picture thresholding using the entropy of the histogram". Signal Processing, vol.2, pp. 223237, 1980.

[4] J. N. Kapur, P. K. Sahoo, K. C. Wong, "A new method for graylevel picture thresholding using the entropy of the histogram”.Computer Vision, Graphics, and Image Processing, vol.29, pp. 273-285, 1985.

[5] A. H. Abutaleb, "Automatic thresholding of gray-level pictures using two-dimensional entropy". Computer Vision, Graphics and Image Processing, vol.47, pp. 22-32, 1989.

[6] M“uller H, Michoux N, Bandon D, Geissbuhler A. A review of contentbased image retrieval systems in medical applications clinical benefits and future directions. Int J Med Inform 2004;73:1-23.

[7] Wong TC. Medical image databases. New York: Springer-Verlag; 1998

[8] TangLHY, Hanka R, Ip HSS.Areviewof intelligent content-based indexing and browsing of medical images. J Health Inform 1999;5:40-9.

[9] Tagare HD, Jaffe CC, Duncan J. Medical image databases: a content-based retrieval approach. J Am Med Informat Assoc 1997;4:184-98.

[10] Florea F,M“uller H, Rogozan A, Geissbuhler A, Darmoni S. Medical image categorization with MedIC and MedGIFT. Proc Med Inform Europe (MIE 2006). p. 3-11.

[11] Lehmann TM, G̈uld MO, Thies C, Fischer B, Keysers D, Kohnen M. Content-based image retrieval in medical applications for picture archiving and communication systems. Proc SPIE 2003;5033:109-17.

[12] El-Kwae EA, Xu H, Kabuka MR. Content-based retrieval in picture archiving and communication systems. IEEE Trans Knowledge Data Eng 2000;13(2):70-81.

[13] G uld MO, Kohnen M, Schubert H, Wein BB, Lehmann TM. Quality of DICOM header information for image categorization. Proc SPIE 2002;4685:280-7.

[14] Smeulders AWM, Worring M, Santini S, Gupta A, Jain R. Content-based image retrieval at the end of the early years. IEEE Trans Pattern Anal Mach Intell 2000;22(12):1349-80.

[15] Rui Y, Huang TS, Chang SF. Image retrieval: current techniques, promising directions and open issues. J Vis Commun Image Rep 1999;10(4):39-62.

[16] M“uller H, Deselaers T, Lehmann TM, Clough P, Kim E, HershW. Overview of the ImageCLEFmed 2006 medical retrieval and annotation Tasks. In:Evaluation of multilingual and multi-modal information retrieval - seventh workshop of the cross-language evaluation forum (CLEF 2006); 2007. ProcLNCS 2006;4730:595-608.

[17] Shyu CR, Brodley CE, Kak AC, Kosaka A, Aisen AM, Broderick LS. ASSERT: a physician-in-the-loop content-based image retrieval system for HRCT image databases. Comput Vis Image Understand 1999;75:111-32.

[18] Long LR, Thoma GR. Landmarking and feature localization in spine Xrays. J Elect Imaging 2001;10(4):939-56. 
[19] Orphanoudakis SC, Chronaki C, Kostomanolakis S. $I 2 C$ - a system for the indexing, storage and retrieval of medical images by content. Med Inform 1994;19(2):109-22.

[20] Mojsilovic A, Gomes J. Semantic based image categorization, browsing and retrieval in medical image databases. Proc IEEE Int Conf Image Process 2002;3:145-8

[21] Lehmann TM, G̈uld MO, Deselaers T, Keysers D, Schubert H, Spitzer K. Automatic categorization of medical images for contentbased retrieval and data mining. Comput Med Imaging Graph 2005;29:143-55.

[22] Vapnik V. Statistical learning theory. New York, NY: Wiley; 1998.

[23] Chapelle O, Haffner P, Vapnik V. SVMs for histogram-based image classification. IEEE Trans Neural Networks 1999;10(5):105564.

[24] Krebel U. Pairwise classification and support vector machines. In: Adv in kernel methods: support vector learning.Cambridge, MA: MIT Press; 1999. p. 255-68.

[25] Chang SF, Sikora T, Puri A. Overviewof the MPEG-7 standard. IEEE Trans Circ Syst Video Technol 2001;11:688-95.

[26] Baeza-Yates R, Ribiero-Neto B. Modern information retrieval. Boston, MA: Addison-Wesley; 1999 